\title{
COMMENTARY
}

\section{Lymph node dissection in radical prostatectomy: What nodes?}

\author{
Stephen Pautler, MD FRCSC \\ Division of Urology, Department of Surgery and Division of Surgical Oncology, Department of Oncology, University of Western Ontario, London, ON
}

See related article on page 254 .

Cite as: Can Urol Assoc J 2011;5(4):260; D01:10.5489/cuaj.11145

$\mathrm{T}$ he subject of pelvic lymphadenectomy for prostate cancer remains a field of intense interest in uro-oncology. While most surgeons agree that lymphadenectomy for staging is not a perfect science, surgical staging remains our best modality. There are many unanswered questions surrounding lymph node dissection such as:

- When to perform a lymph node dissection?

- Do all patients need a node dissection or only intermediate and high-risk individuals?

- Which nodes should be removed and when?

- At what price with respect to complications?

- Can improvements in imaging aid surgeons in targeting at risk nodes?

- Can the node dissection in prostate cancer impact survival?

The authors have attempted to answer the question of the primary landing zone for prostatic lymphatic drainage using the injection of Indocyanine Green dye. ${ }^{1}$ This technique has been used in other cancers with high degree of accuracy and the application to prostate cancer in this small series provides a proof-of-principle. From their results, it appears that the contemporary lymph node dissection (obturator and external iliac nodes) might miss metastatic deposits. We must be cautious with the results based on the small number of patients in this study. Additionally, the low lymph node counts from the internal iliac dissections found in this study (6 of 28 dissections had no nodes), raises some question about the utility of expanding the standard dissection in all patients. Interestingly, the lymph nodes that were positive did not light up using their dye technique due to complete lymphatic obstruction from the cancer cells. They correctly state that this could lead to false negatives if surgeons only targeted dye-containing nodes. Yet another point of caution to consider.

In another recent study using radionuclide injection in the prostate with SPECT/CT and SPECT/MRI followed by surgical mapping, two thirds of primary landing zone nodes were found to be outside of the standard dissection. ${ }^{2}$ Studer's group advocates dissection not only of the external and internal iliac nodes and obturator nodes, but also the common iliac nodes up to the ureter. This has not been standard, but further study is necessary to duplicate these results and to determine if extended node dissections are actually therapeutic.

The controversies in this field are ripe for a prospective study. Level 1 evidence remains our gold standard, yet it is lacking for many of our surgical approaches. While it could take years to obtain survival outcomes, the use of surrogates, such as biochemical progression and selection of high-risk patients only, could facilitate an adequately powered study. Until we take on these challenges, we may never obtain the answers to these fundamental questions.

Competing interests: None declared.

This paper has been peer-reviewed.

\section{References}

1. Inoue S, Shiina $\mathrm{H}$, Arichi $\mathrm{N}$, et al. Identification of lymphatic pathway involved in the spreading of prostate cancer by fluorescence navigation approach with intraoperatively injected indocyanine green. Can Urol Assoc J 2011;5:254-9; D01:10.5489/cuai.10159.

2. Mattei A, Fuechsel FG, Bhatta Dhar N, et al. The template of the primary lymphatic landing sites of the prostate should be revisited: results of a multimodality mapping study. Eur Urol 2008;53:118-25.

Correspondence: Dr. Stephen E. Pautler, Division of Urology, St. Joseph's Hospital, 268 Grosvenor St., London, ON N6A 4V2; fax: 519-646-6037; stephen.pautler@sihc.london.on.ca 